\title{
Unsuspected pheochromocytoma: Is it time for a registry?
}

\author{
Julian Mackenzie-Feder, BScH $\cdot$ Janius Tsang, MD • \\ Sebastian Demyttenaere, MD
}

Received: 3 June 2011/ Accepted: 19 September 2011/Published online: 12 October 2011

(C) Canadian Anesthesiologists' Society 2011

\section{To the Editor,}

Pheochromocytomas are rare catecholamine-secreting tumours that may present at induction of anesthesia. ${ }^{1} \mathrm{~A}$ family history or a history of classic symptoms (headaches, tachycardia, diaphoresis) may be helpful in some patients, but presentations are extremely variable and classic symptoms are seldom all present. Therefore, a high index of suspicion is required to diagnose a pheochromocytoma correctly.

The patient gave consent for publication of the following case. A 70-yr-old male with a history of hypertension and atrial fibrillation was scheduled for laparoscopic bilateral inguinal hernia repair. He had no problems with previous surgeries, and he was not aware of any family history of complications associated with anesthesia. Induction of anesthesia was achieved with sufentanil $15 \mu \mathrm{g}$ $i v$ and propofol $200 \mathrm{mg} i v$, followed by rocuronium $50 \mathrm{mg}$ $i v$. At this point, the patient's heart rate increased to 150 beats. $\min ^{-1}$ with a narrow-complex tachycardia. The trachea was then intubated, and the next blood pressure measurement showed severe hypertension (200/ $110 \mathrm{mmHg}$ ). Despite additional boluses of propofol and sufentanil initially and esmolol later, the patient's blood pressure remained labile. After receiving labetalol, the patient became bradycardic and hypotensive, and he required resuscitation according to Advanced Cardiac Life Support guidelines. He was subsequently transferred to the intensive care unit without undergoing surgery.

Subsequently, a computed tomography scan and magnetic resonance imaging of the patient's abdomen revealed

J. Mackenzie-Feder, BScH $\cdot$ J. Tsang, MD .

S. Demyttenaere, MD $(\bowtie)$

McGill University, Saint Mary’s Hospital, Montreal,

QC, Canada a $3.3 \mathrm{~cm} \times 2.8 \mathrm{~cm}$ heterogeneous mass of the right adrenal gland suspicious for pheochromocytoma (Figure). Biochemical testing confirmed the presence of an epinephrinesecreting pheochromocytoma. The patient was discharged and subsequently underwent a laparoscopic right adrenalectomy after appropriate alpha and beta blockade. The subsequent induction and intraoperative course were uncomplicated. The pathology of the adrenal mass revealed a well-circumscribed $2.5-\mathrm{cm}$ pheochromocytoma.

This case reinforces the notion that pheochromocytomas should be suspected in cases of perioperative hypertensive episodes. This occurrence can easily be overlooked because pheochromocytomas are rare tumours with a reported incidence of $1.5-2$ per million per year. ${ }^{2}$ Furthermore, the presentation can be so varied ${ }^{3}$ that delayed diagnosis is common and has resulted in death in up to $80 \%$ of cases discovered intra- or postoperatively.

Rocuronium was one of the agents given at induction in this case and, based on the literature and the similarity of this event to previous case reports, ${ }^{4}$ it is conceivable that it may have been the precipitating factor. Other than the drugs themselves, pain (related to intravenous drug injection of propofol or tracheal intubation) is another stimulus that has been implicated in the increase in sympathetic activity resulting in a pheochromocytoma crisis. ${ }^{5}$

Rather than discuss treatment options, this report serves to highlight that pheochromocytomas presenting in the operating room seem to be more common than once believed. We suggest a thorough review of pheochromocytoma case reports and the creation of an international registry for pheochromocytoma cases similar to one that exists for malignant hyperthermia. Establishing such a registry would allow for a more comprehensive understanding of these rare tumours. With these data available, it might also be possible to identify these patients more 


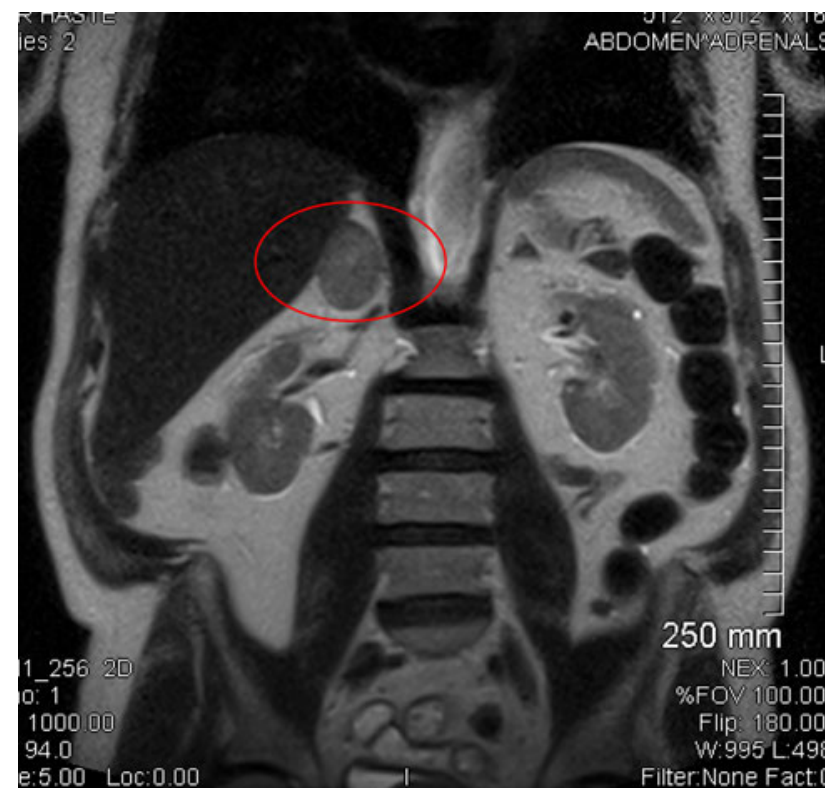

Figure Magnetic resonance imaging coronal section showing a $3.3 \mathrm{~cm} \times 2.8 \mathrm{~cm}$ right adrenal mass

readily in the preoperative setting and to intervene accordingly. Until then, appropriate management of suspected cases and high indices of suspicion are critical in order to minimize the morbidity and mortality associated with manifestations of a pheochromocytoma during anesthesia and surgery.

\section{Competing interests None declared.}

The authors have no disclosures.

\section{References}

1. James $M F$, Cronje $L$. Pheochromocytoma crisis: the use of magnesium sulfate. Anesth Analg 2004; 99: 680-6.

2. O'Riordan JA. Pheochromocytomas and anesthesia. Int Anesthesiol Clin 1997; 35: 99-127.

3. Wooster DL, Mitchell RI. Unsuspected phaeochromocytoma presenting during surgery. Can Anaesth Soc J 1981; 28: 471-4.

4. Holldack HJ. Induction of anesthesia triggers hypertensive crisis in a patient with undiagnosed pheochromocytoma: could rocuronium be to blame? J Cardiothorac Vasc Anesth 2007; 21: 858-62.

5. Morishima $T$, Sobue $K$, Arima $H$, et al. Profound pain due to propofol injection triggered myocardial ischemia in a patient with a suspected pheochromocytoma. Anesth Analg 2003; 96: 631. 